\title{
Hiring Procedures to Implement Stable Allocations*
}

\author{
José Alcalde \\ Dep. Fonaments de l'Anàlisi Econòmica, University of Alicante, E-03071 Alicante, Spain \\ alcalde@merlin.fae.ua.es \\ David Pérez-Castrillo \\ Dep. de Economía e Historia Económica, Universitat Autònoma de Barcelona, \\ 208193 Bellaterra, Barcelona, Spain \\ dpcastrillo@cc.uab.es \\ and \\ Antonio Romero-Medina \\ Dep. de Economía, Universidad Carlos III, 8903 Getafe, Madrid, Spain \\ aromero@eco.uc3m.es
}

\begin{abstract}
We implement the stable correspondence of a job matching market in Subgame Perfect Equilibrium. We use a simple sequential mechanism in which firms propose a salary to each worker (first stage) and, then, each worker accepts at most one proposal (second stage). Moreover, if agents' preferences are additive, this mechanism implements in Subgame Perfect Equilibrium the firms' optimal correspondence when firms use undominated strategies. Finally, we construct another simple sequential mechanism where the order of decisions is permuted and which implements the workers' optimal correspondence when agents' preferences are additive. Journal of Economic Literature Classification Numbers: C78, D78.
\end{abstract}

\section{INTRODUCTION}

In this paper, we provide two mechanisms to implement the stable correspondence (or a proper selection from it) in a job matching market

* We are grateful to Salvador Barberà, Carmen Beviá, Roberto Serrano and an anonymous referee for helpful comments. Our work is partially supported by DGCYT, projects PB 92-0590 and PB 94-1504, and Institut Valencià d'Inverstigacions Econòmiques. RomeroMedina also acknowledges financial support from the Ministerio de Educación y Cultura (Spain). 
with monetary transfers. The mechanisms proposed are very simple. They are two-stage mechanisms. In the first stage, the agents on one side of the market make simultaneous proposals to the members of the other side. In the second stage, once all the proposals have been made, they are either accepted or rejected. The final matching is determined by the second-stage decisions, while the salaries come out from the first-stage proposals.

In "firms go fishing," the first hiring mechanism that we provide, firms play first. Each firm proposes the wage for which it is ready to hire each worker. In the second stage, each worker selects one among the first stage proposals. In a general framework, this mechanism implements the core correspondence in pure strategy subgame perfect Nash equilibrium (SPE). Moreover, if agents' preferences are additive, this mechanism implements in SPE the firms' optimal stable correspondence when firms use undominated pure strategies.

In the second mechanism, "workers go fishing," each worker proposes a firm and the wage for what she is ready to be hired by this firm. Then, each firm selects the set of workers from among those that have chosen that firm at stage one. Assuming that the preferences of the agents are additive, this mechanism implements the workers' optimal stable allocations in SPE.

As it is apparent from the description of the mechanisms, they mimic simple hiring procedures. In this sense, the paper can also be viewed as an analysis of the behavior of simple hiring mechanisms. We show that simple procedures do a very good job. In particular, the outcome of the hiring procedures is stable, therefore the final matching is Pareto efficient.

There is a long tradition of game theoretic analysis in many-to-one matching markets (see Roth and Sotomayor [8] for an excellent survey of the results in matching models up to 1990). In job matching markets, Kelso and Crawford [5] show that stable allocations may fail to exist. This means that, in these markets, the core may be empty. They also provide a property, called the gross-substitute condition, under which the set of stable allocations is non-empty.

To our knowledge, ours is the first paper which deals with the implementation of stable allocations in job markets. Similar questions are being investigated in related economic environments. For the college admissions problems, that is, for many-to-one matching models without monetary transfers, Kara and Sönmez [4] provide a general analysis of the problem of implementation. In particular, they show that the stable correspondence is implementable in Nash equilibrium, while no particular selection from the core is Nash implementable. Also in the college admissions problem, Alcalde and Romero-Medina [2] implement the core correspondence in SPE. They use a sequential mechanism where the colleges (or the students) propose a matching, and then the students (or the colleges) must simply 
accept or reject the proposed allocation. Peleg $[6]^{1}$ considers a very similar sequential mechanism for the marriage (one-to-one matching) problem. ${ }^{2}$ By contrast, in our "firms go fishing" mechanism firms propose prices at which transactions can be made, and then the actual matching is decided by each worker facing all the transaction prices.

Finally, in a context where the bilateral structure plays no role, PérezCastrillo [7] and Serrano [10] present sequential mechanisms which implement the core of any cooperative game in characteristic form.

The paper is organized as follows. Section 2 introduces the basic model. Section 3 presents a mechanism to implement the core for job markets when monetary transfers are allowed. Section 4 studies implementation of both the firms' and workers' optimal stable correspondences in additive environments.

\section{THE MODEL}

We consider a job market with $n$ workers and $l$ firms. Let $W=$ $\left\{w_{1}, \ldots, w_{n}\right\}$ and $F=\left\{f^{1}, \ldots, f^{l}\right\}$ be the set of workers and firms, respectively. The preferences of each worker depend on two variables. The first one is the firm she is working for, whereas the second relevant aspect is the wage that this firm pays to her. Worker $w_{i}$ pr $\mathrm{f} \mathrm{r} \mathrm{nc}$ ar $\mathrm{r}$ pr ntabl by the utility function $U_{i}\left(f^{j}, p_{i}\right)$, which is non-decreasing and continuous in $p_{i} \in \mathbb{R}$, where $p_{i}$ is the salary that worker $w_{i}$ receives and $f^{j}$ is the firm she is working for. A worker who is not hired by any firm reaches a utility level $U_{i}(\varnothing, 0)$. That is, we represent by $f=\varnothing$ the situation in which the worker is not hired by any firm. We also assume that for each firm $f^{j}$ there is a reservation salary $r_{i}^{j}$ such that $U_{i}\left(f^{j}, r_{i}^{j}\right)=U_{i}(\varnothing, 0)$ and that $U_{i}\left(f^{j}, p_{i}\right)$ has the same limit as $p_{i}$ tends to $+\infty$ independently of the identity of the firm $f^{j}{ }^{3}$

The profit of each firm depends on the set of workers it hires, say $W^{j} \subseteq W$, and the salaries it pays to them, say $P^{j}=\left(p_{i}^{j}\right)_{w_{i} \in W^{j}}$. For notational convenience, we will sometimes treat $P^{j}$ as a vector in $\mathbb{R}^{n}$, $P^{j}=\left(p_{i}^{j}\right)_{w_{i} \in W}$, and assume that the profit of the firm $j$ does not depend on $p_{i}^{j}$ for $w_{i} \notin W^{j}$. Let $\pi^{j}: 2^{W} \times \mathbb{R}^{n} \rightarrow \mathbb{R}$ be the profit function of firm $f^{j}$. $\pi^{j}\left(W^{j}, P^{j}\right)$ is decreasing in salaries $p_{i}^{j}$ whenever $w_{i} \in W^{j}$. A firm which does not hire any worker obtains $\pi^{j}(\varnothing, 0)$.

We describe a job market allocation by means of two variables. The first one is a vector $P \in \mathbb{R}^{n}$ representing the wage that each worker gets, where

\footnotetext{
${ }^{1}$ We thank an anonymous associated editor for bringing this paper to our attention.

${ }^{2}$ Peleg [6] also provides a simultaneous mechanism to implement the core in strong equilibrium.

${ }^{3}$ This condition can be relaxed by assuming that the maximum limit of $U_{i}\left(f^{j}, p_{i}\right)$ as $p_{i}$ tends to $+\infty$ is reached by at least two firms.
} 
$p_{i}=0$ if $w_{i}$ is not hired by any firm. The second one is a correspondence, to be called a matching, that states which firm (if any) hires each worker and vice-versa. More precisely, a matching $\mu$ is a correspondence that maps $W \cup F$ into itself such that $(a)$ for each $w_{i} \in W$, if $\mu\left(w_{i}\right)$ does not belong to $F$, then it is the empty set; $(b)$ for each $f^{j}$ in $F, \mu\left(f^{j}\right)$ is contained in $W$, and (c) for each pair $\left(w_{i}, f^{j}\right) \in W \times F, \mu\left(w_{i}\right)=f^{j}$ if and only if $w_{i}$ belongs to $\mu\left(f^{j}\right)$.

We are interested in the job market allocations that are stable. The stability of an allocation depends on the possibilities which agents have to improve their utility level (if workers) or their profits (if firms). An allocation is stable if the following two conditions are satisfied. The first one is individual rationality: Each agent weakly prefers the payoff that she/it gets in this allocation rather than being unmatched. The second one is collective rationality, in the following sense: It is not possible for a firm and a group of workers to allocate their resources, in such a way that both the firm and the workers it hires find the new situation profitable. That is, $(\mu, P)$ is stable if, and only if, $\nexists\left(\hat{W}, f^{j}\right) \in 2^{W} \times(F \cup \varnothing)$ and $\hat{P} \in \mathbb{R}^{n}$ such that

$$
U_{i}\left(f^{j}, \hat{p}_{i}\right)>U_{i}\left(\mu\left(w_{i}\right), p_{i}\right) \text { for all } w_{i} \in \hat{W}, \text { where } \hat{p}_{i}=0 \text { if } f^{j}=\varnothing,
$$

and

(ii) $\pi^{j}(\hat{W}, \hat{P}) \quad \pi^{j}\left(\mu\left(f^{j}\right), P\right)$, wher $\hat{P} \quad 0$ if $\hat{W} \quad \varnothing$

Notice that, in this model, the notion of stability is equivalent to the usual definition of the core. An allocation is stable if it is robust to deviations by a coalition of one firm and a group of workers, or by a firm or a worker alone. These coalitions are the only essential coalitions in this environment. A coalition formed by several firms and workers can be broken down into subunits, each containing at most one firm. Similarly, coalitions formed only by firms or workers can be broken down into subunits each one containing only one agent.

In job markets, the set of stable allocations may be empty. This can happen when workers are complementary in the sense that a set of workers generates more income than the sum of the income from each worker separately (see Roth and Sotomayor [8] for an example). Kelso and Crawford [5] propose a condition, the "gross substitutes condition", that rules out this possibility, guarantying the existence of stable allocations in the market. The assumption requires that increases of other workers' salary should never lead a firm to withdraw an offer from a worker whose salary has not risen. ${ }^{4}$

\footnotetext{
${ }^{4}$ More precisely, denote $M^{j}(P)$ the set of solutions to the problem $\max _{\hat{W} \subset W} \pi^{j}(\hat{W}, P)$. Consider two vectors of salaries $P$ and $\bar{P}$, and a set of workers $\tilde{W} \subseteq W$. Let denote $T^{j}(\tilde{W} ; P, \bar{P}) \equiv\left\{w_{i} \mid w_{i} \in \tilde{W}\right.$ and $\left.p_{i}=\bar{p}_{i}\right\}$. The gross substitutes condition requires that for every $f^{j}$, if $\hat{W} \in M^{j}(P)$ and $\bar{P} \geqslant P$, then there exists $\bar{W} \in M^{j}(\bar{P})$ such that $T^{j}(\hat{W} ; P, \bar{P}) \subseteq \bar{W}$.
} 


\section{THE “FIRMS GO FISHING” MECHANISM}

This section presents a mechanism implementing the stable correspondence in SPE (recall that we only consider pure strategy equilibria). This mechanism reflects a natural form of firms' competition for workers. Firms' behavior will be modeled by a kind of Bertrand competition.

Consider the following two-stage mechanism, called $\Gamma^{F}$. In the first stage each firm proposes a vector of salaries (one for each worker). Once the salaries have been announced, each worker selects a firm. The outcome of this game is the following. The matching is induced by the choices of the workers at the second stage, whereas the salary to be payed is the one that firms proposed at the first stage.

More precisely, at the first stage, firms send their messages simultaneously. Each firm's message space is $\mathbb{R}^{n}$. A message for firm $f^{j}$, $m^{j}=\left(m_{1}^{j}, \ldots, m_{n}^{j}\right)$, will be understood as the salary at which it is willing to hire each worker. At the second stage, and knowing firms' messages, worker $w_{i} \mathrm{~m} \quad$ ag $, m_{i}, \mathrm{i}$ an lem nt of $F \cup\{\varnothing\}$ That i, a work $\mathrm{r}$ trat gy i a function from $\mathbb{R}^{n}$ to $F \cup\{\varnothing\}$ A m ag $m_{i}$ will b under stood as the firm worker $w_{i}$ is willing to work for at the salary proposed by this firm at the first stage. The outcome function $\phi^{1}(\cdot)$ associates to each set of messages, $\tilde{m}=\left(m^{1}, \ldots, m^{j}, \ldots, m^{l}, m_{1}, \ldots, m_{i}, \ldots, m_{n}\right)$ a matching, $\mu^{\tilde{m}}$, and a vector of salaries, $P(\tilde{m}) \in \mathbb{R}^{n}$ such that

(a) for any $w_{i} \in W, \mu^{\tilde{m}}\left(w_{i}\right)=m_{i}$, and

(b) $p_{i}(\tilde{m})=m_{i}^{\mu^{\tilde{m}}\left(w_{i}\right)}$ if $\mu^{\tilde{m}}\left(w_{i}\right) \in F$ and $p_{i}(\tilde{m})=0$ otherwise.

The next theorem analyzes the SPE of the mechanism $\Gamma^{F}$.

THEOREM 3.1. Suppose there are at least two firms. The mechanism $\Gamma^{F}$ implements in SPE the stable correspondence.

Proof. We first prove that each SPE outcome is stable. Let $\tilde{m}$ be the vector of messages that agents state at a certain SPE. Suppose that $\phi^{1}(\tilde{m})=\left(\mu^{\tilde{m}}, P(\tilde{m})\right)$ is not stable. Then we have three possibilities. First, a worker $w_{i}$ can get less utility than $U_{i}(\varnothing, 0)$. In this case, the worker would be better off with the message $m_{i}=\varnothing$ than with $\tilde{m}_{i}$. Second, a firm $f^{j}$ profits can be lower than $\pi^{j}(\varnothing, 0)$. Then, the firm would benefit changing its message $\tilde{m}^{j}$ by $m_{i}^{j}=-\infty$ for all $w_{i} \in W{ }^{5}$ The third possibility is that there is a profitable deviation concerning one firm and a proper subset of workers. Thus, assume that there are a firm $f^{k}$, a set of workers $W^{k}$, and a vector of salaries $\bar{P}$, such that

\footnotetext{
${ }^{5}$ If the reservation salaries $r_{i}^{j}$ are all positive, we do not need negative salaries. For example $m_{i}^{k}=0$ works as well as $m_{i}^{k}=-\infty$.
} 
(i) $U_{i}\left(f^{k}, \bar{p}_{i}\right)>U_{i}\left(\mu^{\tilde{m}}\left(w_{i}\right), p_{i}(\tilde{m})\right)$ for all $w_{i}$ in $W^{k}$, and

(ii) $\pi^{k}\left(W^{k}, \bar{P}\right)>\pi^{k}\left(\mu^{\tilde{m}}\left(f^{k}\right), P(\tilde{m})\right)$.

Let us consider the following strategy for $f^{k}: m_{i}^{k}=\bar{p}_{i}$ if $w_{i} \in W^{k}$ and $m_{i}^{k}=-\infty$ otherwise, keeping constant the strategies $\tilde{m}^{j}$, for $f^{j} \neq f^{k}$. At the second stage each agent in $W$ chooses her employer in order to maximize her utility level. Because of condition $(i)$ above, the message of any worker $w_{i}$ in $W^{k}$ will be $m_{i}=f^{k}$. Notice that $U_{i}\left(f^{k}, \bar{p}_{i}\right)>U_{i}\left(\mu^{\tilde{m}}\left(w_{i}\right), p_{i}(\tilde{m})\right)$ and $U_{i}\left(\mu^{\tilde{m}}\left(w_{i}\right), p_{i}(\tilde{m})\right) \geqslant U_{i}\left(f^{j}, m_{i}^{j}\right)$ for any $f^{j}$ since $\mu^{\tilde{m}}\left(w_{i}\right)$ was the optimal choic giv $\mathrm{n} \tilde{m}$ Mor ov $\mathrm{r}$, no work $\mathrm{r} w_{h}$ out id $W^{k}$ will nd a $\mathrm{m} \mathrm{s}$ ag uch a $m_{h} f^{k}$ Ther for, it $\mathrm{i}$ in $f^{k}$ int $\mathrm{r} \mathrm{t}$ to $\mathrm{d}$ viat which $\mathrm{i}$ a contradiction.

We now prove that each stable allocation can be supported by a SPE. Let $(\mu, P)$ be a stable allocation. Take $w_{i}$ such that $\mu\left(w_{i}\right) \in F$, and denote by $\hat{p}_{i}^{j}$ the salary that firm $f^{j}$ would have to pay to worker $w_{i}$ for her to be indifferent between working in firm $f^{j}$ at a salary $\hat{p}_{i}^{j}$ and working in firm $\mu\left(w_{i}\right)$ at a salary $p_{i}$. Note that $\hat{p}_{i}^{j}$ exists because $U_{i}\left(f^{k}, p_{i}\right)$ has the same limit as $p_{i}$ tends to $+\infty$ independently of $f^{k}$.

Consider the following strategies: Each firm $f^{j}$ sends the message $m^{j}$, where

$$
m_{i}^{j}= \begin{cases}\hat{p}_{j}^{j} & \text { if } \mu\left(w_{i}\right) \in F \\ r_{i}^{j} & \text { otherwise. }\end{cases}
$$

When confronted to the previous firms' strategies, worker $i$ 's message is $m_{i}=\mu\left(w_{i}\right)$. For any other possible firms' strategies, she makes any choice that maximizes her utility. These strategies constitute a SPE yielding the desired allocation.

We can read Theorem 3.1 in two ways. First, the theorem shows that it $\mathrm{i}$ po ibl to implem nt th tabl corr pond nc in quit a $\mathrm{g} \mathrm{n}$ ral mod 1 by $u$ ing $v$ ry simpl $m$ chani $m$ Although $w$ alr ady knew that the stable correspondence (the core) was implementable, it is interesting to know that the implementation can be achieved through "natural" mechanisms. Second, the result tells us that simple hiring procedures do a good job. They lead to stable, and hence efficient, allocations. In this sense, our theorem can be understood as the characterization of the outcome of a particular hiring mechanism.

Theorem 3.1 establishes the equivalence between the core of the job matching market and the SPE of $\Gamma^{F}$. However, we have already remarked that the core may be empty. When this happens, we know that $\Gamma^{F}$ has no SPE in pure strategies. Unfortunately, analyzing mixed strategies of $\Gamma^{F}$ is very difficult, so we cannot characterize the outcome of the mechanism in those games where the stable correspondence is empty. 


\section{4. "FISHING" IN ADDITIVE ENVIRONMENTS}

Sometimes, we would like to have the possibility of selecting some particular allocations from the correspondence of stable allocations. Hence, this section is devoted to study the implementation of particular selections of the stable correspondence. More precisely, we show first that, if firms employ undominated strategies, the mechanism $\Gamma^{F}$ implements in SPE the firms' optimal stable correspondence. Second, we design a mechanism implementing the workers' optimal stable correspondence in SPE. However, in order to do it, we are forced to restrict attention to additive environments: outside these environments, the results do not hold. Let us first recall what an additive environment is, and the characteristics of the two abovementioned stable selections: the firms' and the workers' optimal stable correspondences.

\subsection{Additive Job Matching Markets}

We assume that worker $w_{i}$ pr fer nc ar $\mathrm{r}$ pr s ntabl by the utility function $U_{i}\left(f^{j}, p_{i}\right) \quad p_{i} \quad r_{i}^{j}$, wher $r_{i}^{j} \mathrm{i}$ the $\mathrm{r}$ rvation alary of work $\mathrm{r} w_{i}$ when she is working for firm $f^{j}$. We also assume that there is a function $g^{j}: 2^{W} \rightarrow \mathbb{R}$ which represents the income that firm $f^{j}$ earns whenever it engages a certain set of workers. Thus, if firm $f^{j}$ hires workers in $W^{j}$ at salaries $P^{j}=\left(p_{i}^{j}\right)_{w_{i} \in W^{j}}$, its profit is $\pi^{j}\left(W^{j}, P^{j}\right)=g^{j}\left(W^{j}\right)-\sum_{w_{i} \in W^{j}} p_{i}^{j}$. Moreover, we also assume that it is possible to identify the value of a worker to a firm, this value being independent of the worker's colleagues. That is, there are numbers $g_{i}^{j}$, for $i=1, \ldots, n$ and $j=1, \ldots, m$ such that $g^{j}\left(W^{j}\right)=\sum_{w_{i} \in W^{j}} g_{i}^{j}$. Therefore, $\pi^{j}\left(W^{j}, P^{j}\right)=\sum_{w_{i} \in W^{j}}\left(g_{i}^{j}-p_{i}^{j}\right)$. With this assumption, we rule out the possibility of complementarity among workers.

Given our's hypotheses on workers' utility functions and on firms' profits, the market satisfies the gross-substitutes condition of Kelso and Crawford [5]. Therefore, stable allocations always exist. Moreover, they are easy to characterize. An allocation $(P, \mu)$ is stable if and only if

$$
\begin{aligned}
& \mu\left(w_{i}\right)=f^{j} \Rightarrow p_{i}-r_{i}^{j} \geqslant \max \left\{\left\{g_{i}^{k}-r_{i}^{k}\right\}_{k \neq j}, 0\right\} \quad \text { and } p_{i} \leqslant g_{i}^{j}, \\
& \mu\left(w_{i}\right)=\varnothing \Rightarrow \max _{k}\left\{g_{i}^{k}-r_{i}^{k}\right\} \leqslant 0 .
\end{aligned}
$$

Notice that the set of stable matchings coincides with the set of efficient and individually rational matchings. Among the set of stable allocations, it is possible to identify the subset of allocations most preferred by firms. This is the firms' optimal stable correspondence. For a stable allocation to be firms' optimal, the necessary and sufficient condition is that

$$
\mu\left(w_{i}\right)=f^{j} \in F \Rightarrow p_{i}=\max \left\{\left\{g_{i}^{k}-r_{i}^{k}+r_{i}^{j}\right\}_{k \neq j}, r_{i}^{j}\right\} .
$$


Finally, the necessary and sufficient condition for a stable allocation to be workers' optimal is

$$
\mu\left(w_{i}\right)=f^{j} \in F \Rightarrow p_{i}=g_{i}^{j} .
$$

\subsection{Firms' Fishing}

In order to introduce the next result, let us consider the following very simple market. There are two firms $f^{1}$ and $f^{2}$ and one worker, with $g_{1}^{1}=5$, $g_{1}^{2}=2$, and $r_{1}^{1}=r_{1}^{2}=0$. The stable allocations match the worker with $f^{1}$ at a salary $p \in[2,5]$. Consider the stable allocation with $p=4$. The only strategy-profile that supports this allocation in the mechanism $\Gamma^{F}$ is the following: $m^{1}=m^{2}=4$ and

$$
m_{1}= \begin{cases}f^{1} & \text { if } m^{1} \geqslant m^{2} \text { and } m^{1} \geqslant 0 \\ f^{2} & \text { if } m^{2}>m^{1} \text { and } m^{2} \geqslant 0 \\ \varnothing & \text { otherwise. }\end{cases}
$$

However, note that the strategy $m^{2}=4$ is weakly dominated for firm two by the strategy $m^{2}=2$. That is, the SPE implementation of the stable correspondence requires the use of dominated strategies.

We say that a SPE is an undominated SPE (USPE) if no agent plays a strategy which is dominated for her/it. Theorem 4.1 analyzes the outcome of the mechanism $\Gamma^{F}$ in additive environments when firms use undominated strategies.

THEOREM 4.1. In additive environments the mechanism $\Gamma^{F}$ implements in USPE the firms' optimal stable correspondence.

Proof. By Theorem 3.1 we know that every SPE outcome is stable. Let $\tilde{m}$ be the vector of messages that agents state in a certain SPE for this game. Assume that $\phi^{1}(\tilde{m}) \quad\left(\mu^{\tilde{m}}, P(\tilde{m})\right)$ do not b long to the firm optimal table corr pondenc $W$ ar going to how that at lea $t$ on firm is using a dominated strategy.

Since $\left(\mu^{\tilde{m}}, P(\tilde{m})\right)$ is stable but is not optimal from the point of view of firms, there should be $w_{i}$ and $f^{j}$ such that

$$
\mu^{\tilde{m}}\left(w_{i}\right)=f^{j} \in F \quad \text { with } \quad p_{i}(\tilde{m})>\max \left\{\left\{g_{i}^{k}-r_{i}^{k}+r_{i}^{j}\right\}_{k \neq j}, r_{i}^{j}\right\} .
$$

Assume that $\max \left\{\left\{g_{i}^{k}-r_{i}^{k}+r_{i}^{j}\right\}_{k \neq j}, r_{i}^{j}\right\}=g_{i}^{h}-r_{i}^{h}+r_{i}^{j}$ for some $h \neq j$ (the other case is similar). Given that the allocation is the outcome of a SPE, it cannot be the case that $p_{i}(\tilde{m})=m_{i}^{j}>m_{i}^{k}-r_{i}^{k}+r_{i}^{j}$ for every $k \neq j$. Otherwise, firm $j$ would have a profitable deviation decreasing $m_{i}^{j}$ while keeping it above $m_{i}^{k}-r_{i}^{k}+r_{i}^{j}$ for every $k \neq j$. Thus, $\exists k \neq j$ such that $p_{i}(\tilde{m})=m_{i}^{k}-r_{i}^{k}+r_{i}^{j}$ and $p_{i}(\tilde{m})>g_{i}^{k}-r_{i}^{k}+r_{i}^{j}$. Then, $m_{i}^{k}>g_{i}^{k}$. But it is a 
dominated strategy for a firm to make offers greater than its valuation, since in the best possible case it does not hire the worker (so it gets zero from this offer), while it could be the case that its offer is the most attractive for the worker an then it loses money with her. The strategy $m_{i}^{k}$ is dominated by $\bar{m}_{i}^{k}=g_{i}^{k}$.

For the reverse implication, let $(\mu, P)$ be a firms' optimal stable allocation. Consider the following strategies. The message (and strategy) of firm $f^{j}$ is $m^{j}=\hat{P}^{j}$, where

$$
\hat{p}_{i}^{j}= \begin{cases}p_{i} & \text { if } w_{i} \in \mu\left(f^{j}\right) \\ g_{i}^{j} & \text { otherwise. }\end{cases}
$$

When confronted with the previous firms' strategies, worker $i$ 's message is $m_{i} \mu\left(w_{i}\right)$ For any other pos ibl firm trat gi, $\mathrm{h}$ mak any choic that maximiz her utility The trategi for firm and work $r$ con titut a SPE leading to $(\mu, P)$. Moreover, no agent uses dominated strategies.

Note that the mechanism $\Gamma^{F}$ generates a sort of Bertrand competition among firms. Nevertheless, as Theorem 4.1 states, the outcomes that are expected from the agents' strategical behavior are the optimal stable allocations from the firms' point of view. Hence, the competition between the firms is strong enough to make them reach stable outcomes, but the fact that they are playing first allows them to reach the most profitable among the stable allocations.

The result that Bertrand competition among firms does not lead to workers' optimal allocations is not surprising in our framework. This can be seen as follows. The firms know that, at the second stage, each worker has a dominant strategy, which consist of selecting the firm with whom she maximizes her utility. Given this, firms can obtain the maximum benefit from strategical behavior. Similar results in matching models are due to Alcalde [1] for one-to-one matching markets and to Schummer [9] and Demange and Gale [3] for the assignment problem. In Serrano [10], where the core of convex games is implemented, the player who acts first, announcing the vector of prices, (the broker, in Serrano's terminology) also gets her best core payoff.

Unfortunately, it is not possible to generalize Theorem 4.1 to more general environments. In markets where the value of a worker to a firm depends on other workers hired by the firm, the mechanism $\Gamma^{F}$ can implement in undominated strategies a set of outcomes larger than the firms' optimal stable allocations.

As a simple example, consider two firms, $f^{1}$ and $f^{2}$, and two workers, $w_{1}$ and $w_{2}$. Workers maximize salary and their reservation salary is zero. Firms profits are given by $\pi^{j}\left(W^{j}, P^{j}\right)=g^{j}\left(W^{j}\right)-\sum_{w_{i} \in W^{j}} p_{i}^{j}$, where: $g^{j}\left(\left\{w_{i}\right\}\right)=3$ and $g^{j}\left(\left\{w_{1}, w_{2}\right\}\right)=4$, for $j, i=1,2$. 
The undominated strategies represented by the messages $m^{1}=m^{2}=$ $(2,2), m_{1}=f^{1}, m_{2}=f^{2}$, with any optimal workers' response for out-ofequilibrium firms' strategies, lead to a stable allocation which is not firms' optimal allocation.

\subsection{Workers' Fishing}

This subsection presents a two-stage mechanism implementing the workers' optimal stable allocation in SPE. Let us refer to it by $\Gamma^{W}$. In the fir $\mathrm{t}$ tag, work $\mathrm{r}$ play imultan ou ly Each of th $\mathrm{m}$ announc a alary and a firm for which $\mathrm{h} i \mathrm{i}$ willing to work Th work $\mathrm{r}$ will only $\mathrm{b}$ hir $\mathrm{d}$ if she is offered at least this salary by the firm. Once the salary demands to the firms have been announced, firms simultaneously (or sequentially) select their set of workers. The outcome is determined as follows. The matching is the one induced by the choice of the firms at the second stage, whereas the salary to be payed is determined by the demands of the workers.

More precisely, each worker's message space is $\mathbb{R} \times\{F \cup \varnothing\}$. A message for worker $w_{i}, m_{i}=\left(p_{i}, f_{i}\right)$, will be understood as the salary $p_{i}$ at which she is willing to work for a certain firm $f_{i}$. Knowing the workers' messages, firm $f^{j}$ 's message, $m^{j}$, is a selection from $\left\{w_{i} \in W \mid f_{i}=f^{j}\right\}$. That is, a firm $f^{j}$ 's strategy is a function from $[\mathbb{R} \times\{F \cup \varnothing\}]^{n}$ to $2^{W}$ such that $m^{j}\left(m_{1}, \ldots, m_{n}\right) \subset\left\{w_{i} \in W \mid f_{i}=f^{j}\right\}$. A message $m^{j}$ will be understood as the set of workers that firm $f^{j}$ is willing to hire given the salaries they asked for at the first stage. The outcome function $\phi^{2}(\cdot)$ associates with each message, $\tilde{m}=\left(m_{1}, \ldots, m_{i}, \ldots, m_{n}, m^{1}, \ldots, m^{j}, \ldots, m^{l}\right)$ a matching, $\mu^{\tilde{m}}$, and a vector of salaries, $P(\tilde{m}) \in \mathbb{R}^{n}$ such that

(a) for each $f^{j} \in F, \mu^{\tilde{m}}\left(f^{j}\right)=m^{j}$, and

(b) $p_{i}(\tilde{m})=p_{i}$ if $\mu^{\tilde{m}}\left(w_{i}\right) \in F$, or $p_{i}(\tilde{m})=0$ otherwise.

We next introduce our main result in this section.

THEOREM 4.2. In additive environments the mechanism $\Gamma^{W}$ implements in SPE the workers' optimal stable correspondence.

Proof. First, a firm $f^{j}$ is willing to satisfy any demand of a worker whose value is above her demand. Therefore, in any SPE, it is the case that $w_{i} \in m^{j}$ whenever $f_{i}=f^{j}$ and $p_{i}<g_{i}^{j}$, while $w_{i} \notin m^{j}$ whenever $p_{i}>g_{i}^{j}$. Second, for a given firm, the worker is interested in maximizing the salary. Let worker $w_{i}$ send the message $m_{i}=\left(p_{i}, f_{i}\right)$ in equilibrium, where $p_{i}=0$ if $f_{i}=\varnothing$. It is necessarily the case that $p_{i}=g_{i}^{f_{i}}$ if $f_{i} \in F$. A message with $p_{i}>g_{i}^{f_{j}}$ would lead to $w_{i}$ remaining unmatched, while if $p_{i}<g_{i}^{f_{j}}$ and $g_{i}^{f_{j}}>r_{i}^{f_{j}}$, then worker $w_{i}$ could increase her demand of salary, still being sure that her offer would be accepted. Finally, worker $w_{i}$ can choose among the set of 
firms or remaining unmatched, knowing the maximum demand that each firm is ready to accept. She will choose the situation that maximizes her utility, that is, in equilibrium it is the case that

$$
\begin{aligned}
& f_{i} \in F \Rightarrow f_{i} \in \underset{k}{\arg \max }\left\{g_{i}^{k}-r_{i}^{k}\right\}, \quad \text { and } \\
& f_{i}=\varnothing \Rightarrow \max _{k}\left\{g_{i}^{k}-r_{i}^{k}\right\} \leqslant 0
\end{aligned}
$$

Moreover, in equilibrium, $p_{i}=g_{i}^{f_{i}}$. Therefore, the equilibrium leads necessarily to a workers' optimal allocation.

On the other hand, let $(\hat{\mu}, \hat{P})$ be a workers' optimal stable allocation. Let us consider the following strategies. For each worker $w_{i}$ her message (strategy) is $m_{i}=\left(p_{i}, f_{i}\right)$, where

$$
\left(p_{i}, f_{i}\right)= \begin{cases}\left(\hat{p}_{i}, \hat{\mu}\left(w_{i}\right)\right) & \text { if } \hat{\mu}\left(w_{i}\right) \in F \quad \text { and } \\ (0, \varnothing) & \text { if } \quad \hat{\mu}\left(w_{i}\right) \notin F .\end{cases}
$$

Each firm $f^{j}$ 's message is

$$
m^{j}=\left\{w_{i} \in W \mid p_{i} \leqslant g_{i}^{j}\right\} .
$$

These strategies yield a SPE whose outcome is the allocation $(\hat{\mu}, \hat{P})$.

Note that, when markets are not additive, unstable allocations can be supported by SPE of the mechanism $\Gamma^{W}$, as shown in the next example. See Fig. 1.

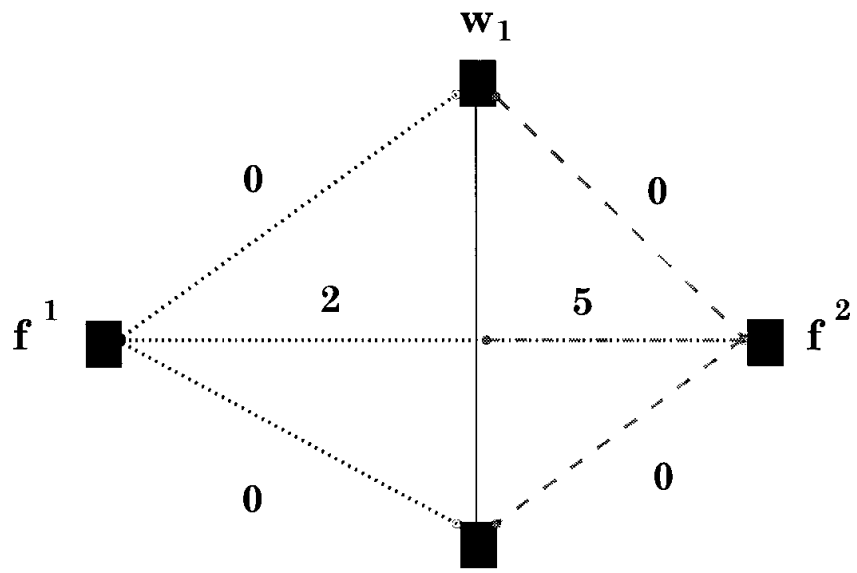

$\mathbf{w}_{2}$

FIGURE 1. 
Let $F=\left\{f^{1}, f^{2}\right\}, \quad W=\left\{w_{1}, w_{2}\right\}, r_{i}^{j}=g^{j}\left(\left\{w_{i}\right\}\right)=0$ for all $i$ and $j$, $g^{1}(W)=2$, and $g^{2}(W)=5$. In such a case, the strategies $m_{1}=m_{2}=\left(f^{1}, 1\right)$, $m^{1}=\left\{w_{1}, w_{2}\right\}$ and $m^{2}=\varnothing$, with any optimal firms' response for out-ofequilibrium workers' strategies, constitute a SPE for $\Gamma^{W}$. Note that the outcome of such an equilibrium is not stable because it can be blocked by $f^{2}$ and $W$ by stating salaries $p_{1}=p_{2}=2$.

Let us finally remark that we can also state Theorem 4.2 in undominated strategies, since we have not used dominated strategies in the proof. That is, the mechanism $\Gamma^{W}$ impl $\mathrm{m} \mathrm{nt}$ in SPE the work $\mathrm{r}$ optimal table allocation when work $r$ trategi ar undominat $\mathrm{d}$

\section{REFERENCES}

1. J. Alcalde, Implementation of stable solutions to marriage problems, J. Econ. Theory 69 (1996), 240-254.

2. J. Alcalde and A. Romero-Medina, "Simple Mechanisms to Implement the Core of College Admissions Problems," IVIE WP-AD 96-13, 1996.

3. G. Demange and D. Gale, The strategy of two-sided matching markets, Econometrica $\mathbf{5 3}$ (1985), 873-888.

4. T. Kara and T. Sönmez, Implementation of college admission rules, Econ. Theory 9 (1997), 197-218.

5. A. S. Kelso and V. P. Crawford, Job matching, coalition formation, and gross substitutes, Econometrica 50 (1982), 1483-1504.

6. B. Peleg, "Implementation of the Core of Marriage Problem," DP 132, Hebrew University of Jerusalem, 1997.

7. D. Pérez-Castrillo, Cooperative outcomes through non-cooperative games, Games Econ. Behav. 7 (1994), 428-40.

8. A. E. Roth and M. Sotomayor, "Two-sided Matching: A Study in Game-Theoretic Modeling and Analysis," Econometric Society Monograph Series, Cambridge Univ. Press, New York, 1990.

9. J. Schummer, "Dominance Solvability in Multi-object Auction," mimeographed, University of Rochester, 1994.

10. R. Serrano, A market to implement the core, J. Econ. Theory 67 (1994), 285-294. 